\title{
Hierarchical Key Establishment Protocols Based on Secure Keyed One-Way Hash Functions
}

\author{
Wei-Chi Ku and Sheng-De Wang \\ Department of Electrical Engineering \\ National Taiwan University, Taipei 106, Taiwan \\ [wcku, sdwang]@star.ee.ntu.edu.tw
}

\begin{abstract}
As key establishment protocols (KEP) are usually the initial step for setting up a secure network-based service, they are very important in enabling the required security. Most of the existing KEPs make use of cryptographic algorithms, either secret key or public-key cryptography. However, it is also possible to employ secure keyed oneway hash functions (SKOWHF) in KEP designs. Though several works have been published in this area, they only focus on the small scale networks. In this paper, we first introduce the SKOWHF-based secure trunks and secure channels; then, two rules for KEP construction, KERI and KER2, are described. Finally, a hierarchical KEP for large scale networks is proposed.
\end{abstract}

\section{Introduction}

Before conducting a secure session using secret-key cryptographic technique, a secret key should be agreed upon by the communicants. To assure strict security, the secret key should be renewed for each session, i.e., it should be a session key, so that compromising one key will not divulge the contents of other sessions. The protocol for establishing a session key between the communicants is referred to as a key establishment protocol (KEP). As KEP is usually the initial process for setting up the secure network-based services, they are crucial in enabling the required security.

To date, the majority of KEPs employ either secret-key cryptosystems or public key cryptosystems. However, it is also possible to use one-way hash functions rather than encryption algorithms to construct KEP. Much research has been published in this area [2]-[8]. Gong [2] presents the original idea of using a one-way hash function as the basic building block of a KEP. Later, Bull, Gong and Sollins [3] propose a KEP also based on one-way hash function. Another well-known instance is Kryptoknight [5][6], which is a family of KEPs developed by IBM. Compared with encryption algorithms, a one-way hash function is easier to be implemented for not having to provide the invertible property [2][5][6]. It also makes the object codes and the source codes of them exportable [2][3][5][6][7][8]. In particular, its computation is less complex [5][6]. Maybe it is the reason why Global System for Mobile Communications (GSM) [1] uses one-way functions in its security system. It has been recognized that the characterization of the one-way hash function specified in the above mentioned works is not appropriate for the way they adopt it in a keyed manner [7][8]. Berson, Gong and Lomas redefine the properties of the suitable one-way hash function for security use, labeled as secure keyed one-way hash function (SKOWHF). In addition, mix-up of confidentiality and authentication usually makes the design more difficult to be analyzed or to be implemented. To solve this problem, Boyd and Mathuria [8] propose a systematic method for KEP construction by sharply distinguishing between the confidentiality and authentication channels.

Most of the existing SKOWHF-base KEPs simply use one trusted key server (or key distribution center) for key establishment. However, since the number of users in the network increases extremely, it is inefficient for a single key server to handle the key establishment [9]. Hence a hierarchical KEP suitable for large scale networks should be contrived. In this paper, we first introduce the basic building blocks, including two secure trunks and three secure channels. Base on these blocks, two approaches to KEP construction are proposed. The first approach provides more flexibility for key generation while the second approach requires fewer computations. Finally, we introduce a hierarchical KEP for large scale and administratively heterogeneous networks, e.g. internet.

\section{Basic building blocks}

For convenience, the definition of SKOWHF, which 
is proposed by Berson, Gong and Lomas [7] is repeated here. A function $f$ is a SKOWHF if it satisfies:

- $f$ maps key $k$ and a bit string $x$ to a output string of fixed length.

- Given $k$ and $x$, it is easy to compute $f(k,\{x\})$.

- Given $k$ and $f(k,\{x\})$, it is computationally infeasible to compute $x$.

- Given $k$, it is computationally infeasible to find two values $x$ and $y(x \neq y)$ such that $f(k,\{x\})=f(k,\{y\})$.

- Given pairs $x$ and $f(k,\{x\})$, it is computationally infeasible to compute $k$.

- Without knowledge of $k$, it is computationally infeasible to compute $f(k,\{x\})$ for any $x$.

- The mapping from $\{k,\{x\}\}$ to $f(k,\{x\})$ is randomly chosen in the sense that it should not be possible to predict any portion of $f(k,\{x\})[8]$.

Some researchers are convinced that an SKOWHF can be constructed by using existing unkeyed hash functions such as MD5 [10], SHA [11] and Snefru [12]. In the rest of this paper, $f$ is used to referred to as an SKOWHF.

\subsection{Secure trunks}

For a sequence of $Z$ nodes $X_{1}, X_{2}, \ldots, X_{Z-1}, X_{Z}$ (not necessary in geographical order), we assume that there exists a common key for each pair of adjacent nodes. That is, $X_{1}$ and $X_{2}$ have shared a common key $C K_{12}, X_{2}$ and $X_{3}$ have shared a common key $C K_{23}, \ldots, X_{Z-I}$ and $X_{Z}$ have shared a common key $C K_{(Z-I) Z}$.

\section{- Confidentiality trunk}

A confidentiality trunk for $m$ from $X_{i}$ to $X_{i+1}(1 \leq i \leq z$ 1) ensures that only the authorized $X_{i+1}$ will be able to read $m$ sent by $X_{i}$ and is denoted by

$$
X_{i}(\mathrm{c}) \rightarrow X_{i+1}: m .
$$

This confidentiality trunk can be realized by using $f$ accompanying with the one-time padding technique in the way as follows:

$$
X_{i} \rightarrow X_{i+1}: n_{X i}, f\left(C K_{X i X i+1},\left\{n_{X i}\right\}\right) \oplus m
$$

where $n_{X i}$ is the nonce issued by $X_{i}$, i.e., this cannot be repeatedly used by $X_{i}$ with the same key as $C K_{X i X i+1}$. By padding with $f\left(C K_{X i X i+1},\left\{n_{X i}\right\}\right), X_{i}$ can send $m$ to $X_{i+1}$ without hesitating that it will be compromised to others. Note that a confidentiality trunk doesn't have to ensure that the messages will certainly be obtained by the expected recipient.

- Integrity trunk

An integrity trunk for $m$ from $X_{i}$ to $X_{i+1}(1 \leq i \leq z-1)$ ensures that $X_{i+1}$ can check that the received $m$, which is sent by $X_{i}$, has never been tampered and is denoted by

$$
X_{i}(\mathrm{i}) \rightarrow X_{i+1}: m
$$

And, this integrity trunk can be realized by using $f$ as below.

$$
X_{i} \rightarrow X_{i+1}: m, f\left(C K_{X i X i+1},\{m\}\right) .
$$

From another view, $X_{i+1}$ can check to see whether $m$ is (or was) actually sent by $X_{i}$. Note that an integrity trunk doesn't have to ensure that the received information is newly sent by the claimed sender. In other words, $X_{i+1}$ doesn't need to verify the freshness of that message.

\subsection{Secure channels}

Now, we employ the proposed two secure trunks to build the secure channels: confidentiality channel, integrity channel and authentication channel. Let $X_{I}$ and $X_{Z}$ represents the end users of the secure channel, in addition, the intermediate nodes between them, i.e., $X_{2}, X_{3}, \ldots, X_{Z-1}$, are trustworthy.

- Confidentiality channel

The confidentiality channel for the message $m$ from $X_{I}$ to $X_{z}$, denoted by

$$
X_{I}(\mathrm{c}) \Rightarrow X_{z}: m \text {, }
$$

can be constructed by orderly cascading the following secure trunks:

$$
\begin{aligned}
X_{I}(\mathrm{c}) & \rightarrow X_{2}: m \text { and } X_{I}(\mathrm{i}) \rightarrow X_{2}:\left\{m, I D_{X z}\right\} \\
& \ldots \\
X_{z-2}(\mathrm{c}) & \rightarrow X_{z-l}: m \text { and } X_{z-2}(\mathrm{i}) \rightarrow X_{z-l}:\left\{m, I D_{X z}\right\} \\
X_{z-l}(\mathrm{c}) & \rightarrow X_{z}: m
\end{aligned}
$$

where $I D_{X z}$ represents the identity of $X_{z}$. Since the intermediate nodes $X_{2}, X_{3}, \ldots$, and $X_{z-1}$ are assumed to be trustworthy, $X_{1}$ can believe that only the authorized user $X_{z}$ will be able to read $m$.

\section{- Integrity channel}

The integrity channel for the message $m$ from $X_{I}$ to $X_{z}$, which is denoted by

$$
X_{l}(\mathrm{i}) \Rightarrow X_{z}: m \text {, }
$$

can be constructed by orderly cascading the following secure trunks:

$$
\begin{aligned}
X_{1}(\mathrm{i}) & \rightarrow X_{2}: m \\
X_{2}(\mathrm{i}) & \rightarrow X_{3}:\left\{m, I D_{X 1}\right\} \\
& \ldots \\
X_{z-1}(\mathrm{i}) & \rightarrow X_{z}:\left\{m, I D_{X 1}\right\} .
\end{aligned}
$$

The integrity channel for $m$ from $X_{I}$ to $X_{Z}$ ensures that $X_{Z}$ can check that $m$ is or has been sent by $X_{l}$. Moreover, each individual item contained in $m$ cannot be modified alone unless the whole $m$ is replaced with an old one ever sent by $X_{1}$.

\section{- Authentication Channel}

The authentication channel for the message $m$ from $X_{I}$ to $X_{z}$, which is dented by

$$
X_{1}(\mathrm{a}) \Rightarrow X_{z}: m,
$$

can be constructed by directly using the integrity channel from $X_{1}$ to $X_{z}$ :

$$
X_{I}(\mathrm{i}) \Rightarrow X_{z}:\left\{m, n_{X z}\right\},
$$

where $n_{X z}$ is a nonce issued by $X_{z}$ and is used as the fresh- 
ness identifier for $m$. From this channel, $X_{z}$ should be able to check the freshness of $m$ and the sender of $m$. If verified, $X_{z}$ can believe that $m$ is newly sent by $X_{l}$.

Remark 1. The assumption of using trustworthy intermediate nodes between $X_{1}$ and $X_{z}$ will be reasonably justified later in the proposed hierarchical KEP, in which these nodes are acted by key servers rather than common users.

\section{Two approaches to KEP construction}

In this section, two approaches to KEP construction are described. The major difference between these two approaches is the manner of generating a session key. In the first approach, the session key is generated by an independent random number generator. While in the second approach, the session key is generated by using an SKOWHF. Mutual confirmation can be achieved in both approaches so that one can check to see whether the peer end has obtained the right session key or not. In fact, Boyd and Mathuria omit such a function for simplification. However, for practical use, mutual confirmation should be considered in the KEP so that the resulting design can serve as a complete module for the adopted system. Notations $A$ (the originator) and $B$ represent the two users who are going to establish a session key.

\subsection{The first approach}

A session key sk, which is independently generated, can be established between $A$ and $B$ using the authentication and confidentiality protocols according to the following key establishment rule.

\section{KER1:}

(1) $A$ sends $n_{A}$ to $B$.

(2) $B(\mathrm{c}) \Rightarrow A:\{s k, y\}$ and $B(\mathrm{a}) \Rightarrow A:\{s k, y\}$

(3) $A \rightarrow B: y$

Nonce $y$ is issued by $B$ and is used for confirming $A$ 's knowledge of $s k$.

Theorem 1. According to KERI, A and B can secretly share sk.

Proof: Since $s k$ is independently generated by $B$, it implies that $B$ believes it is a fresh session key. From the first part of (2), $\{s k, y\}$ can be sent to $A$ confidentially. From the second part of (2), $A$ can make sure that the retrieved $\{s k, y\}$ is newly sent by $B$. If succeeds, $A$ can also believe that $B$ has obtained $s k$. From (3), $B$ can check to see whether the received $y$ is equal to the one previously issued, if so, he believes $A$ has obtained the right $s k$. Consequently, $s k$ is secretly shared by $A$ and $B$.

\subsection{The second approach}

A session key can be generated by computing the session key generation formula (SKGF) rather than by a random number generator. With this approach, some SKOWHF computations can be mustered out.

\section{KER2:}

(1) $A$ sends $n_{A}$ to $B$.

(2) Case I. If $A$ and $B$ have shared a common key $C K_{A B}, B$ computes the SKGF $\left\{s k, h_{A}, h_{B}\right\}=f\left(C K_{A B}\right.$, $\left.\left\{n_{A}, n_{B}\right\}\right)$ and then sends $\left\{n_{B}, h_{B}\right\}$ to $A$. Next, $A$ also computes the same formula as $B$ does.

Case II. If $A$ and $B$ don't share a common key, $B$ computes SKGF $\left\{s k, h_{A}, h_{B}\right\}=f\left(C K_{B W},\left\{n_{A}, n_{B}\right.\right.$, $\left.\left.I D_{A}\right\}\right)$ and then sends $\left\{I D_{A}, n_{A}, n_{B}, h_{B}\right\}$ to his adjacent node $W$. Next, $W$ also computes $\left\{s k, h_{A}, h_{B}\right\}$ as $B$ does. If the computed $h_{B}$ equals the received one, $W(\mathrm{c}) \Rightarrow A:\left\{s k, h_{A}\right\}$ and $W(\mathrm{a}) \Rightarrow A:\left\{s k, h_{A}\right\}$.

(3) $A \rightarrow B: h_{A}$

Handshake numbers $h_{A}$ and $h_{B}$ are used by $A$ and $B$, respectively, for mutual confirmation.

Theorem 2. Following KER2, A and B can secretly share sk.

Proof: In case I, $C K_{A B}$ is only known to $A$ and $B$, so no one else can obtain $\left\{s k, h_{A}, h_{B}\right\}$ using the SKGF. Furthermore, $n_{A}$ and $n_{B}$ are in the input of $f$ and it implies that $\left\{s k, h_{A}, h_{B}\right\}$ is controlled by both $A$ and $B$ rather than by either one alone. On receiving $n_{B}, A$ can compute the SKGF to derive $h_{B}$ and then check to see whether it equals to the received one. If so, he believes that $B$ has obtained the right session key. On receiving $h_{A}$ in (3), $B$ believes that $A$ has obtained the right session key.

In case II, a confidentiality channel and an integrity channel for $\left\{s k, h_{A}, h_{B}\right\}$ from $A$ to $W$ can be constructed. If the $n_{A}$ received by $W$ is fresh, a confidentiality channel and an authentication channel for $\left\{s k, h_{A}\right\}$ from $W$ to $A$ is subsequently constructed. From the characteristic of the authentication channel, a confidentiality channel and an authentication channel for $\left\{s k, h_{A}\right\}$ from $B$ to $A$ is constructed. In the meanwhile, $A$ believes that $B$ has obtained the right session key. On receiving $h_{A}$ in (3), $B$ believes that $A$ has obtained the right session key.

Hence $s k$ can be secretly shared by $A$ and $B$ no matter in case I or II.

\section{Hierarchical key establishment protocols}

For practical use, we use the proposed key establishment rules to construct KEP for users within a large scale network such as Internet [9].

\subsection{Hierarchical structure}

Because the number of network users increases drasti- 


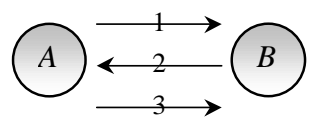

Figure 1. KEP steps of scenario 1.

cally, it is inefficient for a single key server (or key management center) to execute key establishment. To reduce the management complexity, the system can be organized hierarchically [9]. A number of users are under the control of a domain key server and a number of domain key servers are under the control of a cluster key server, and so on. It is assumed that each entity has shared a common key, or mater key, with his control server. To provide greater flexibility, entities of the same level are allowed to share a common key. It is economical when they frequently communicate. Let $A$ and $B$ denote the originator and the corresponding user of the protocol, respectively. $M K_{A}$, the master key of $A$, is used as the common key for $A$ and his key server. Similarly, $M K_{B}$ is the master key of $B$ and is used as the common key for $B$ and his key server.

\subsection{Scenarios}

In the following, we simultaneously apply KER1 and KER2 to construct hierarchical KEPs. Four scenarios are used to illustrate the way of establishing session keys for the most common spots in large scale networks.

Scenario 1. A and B have already shared a common key $C K_{A B}$. (See also Figure 1.)

Involved key servers: none

\section{- According to KER1}

In this situation, $A$ and $B$ can directly construct the needed confidentiality channel and authentication channel without the help of any key server. The KEP is illustrated as follows:

Step 1. $A \rightarrow B: n_{A}$

$B$ generates the session key sk using an independent key generator, e.g., a random number generator. Then, sk combined with another nonce $y$ is transmitted back to $A$ over the secure channels where $f\left(C K_{A B},\left\{n_{B}\right\}\right) \oplus\{s k, y\}$ forms the confidentiality channel and $f\left(C K_{A B},\left\{s k, y, n_{A}\right\}\right)$ forms the authentication channel.

Step 2. $B \rightarrow A: n_{B}, f\left(C K_{A B},\left\{n_{B}\right\}\right) \oplus\{s k, y\}, f\left(C K_{A B},\left\{s k, y, n_{A}\right\}\right)$ Once all the received messages have been verified, $A$ believes that $s k$ is newly sent by $B$. However, $B$ doesn't know that whether $A$ has obtained the correct session key

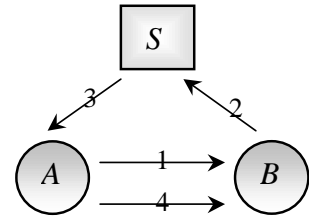

Figure 2. KEP steps of scenario 2.

or not. Accordingly, a further confirmation from $A$ to $B$ should be conducted as follows:

Step 3. $A \rightarrow B: y$

In the protocol, $A$ needs to perform two SKOWHF computations, and so does $B$.

- According to KER2

Clearly, the rule specified in case I of KER2 can be directly used here.

Step 1. $A \rightarrow B: n_{A}$

On receiving $n_{A}, B$ generates $n_{B}$ and then computes the SKGF:

$$
\left\{s k, h_{A}, h_{B}\right\}=f\left(C K_{A B},\left\{n_{A}, n_{B}\right\}\right) .
$$

Next, $B$ sends $n_{B}$ and $h_{B}$ back to $A$.

Step 2. $B \rightarrow A: n_{B}, h_{B}$

The handshake number $h_{B}$ is used by $B$ to inform his knowledge of $s k$ to $A$. After retrieving $n_{B}$ from the received message, $A$ also computes the SKGF. Next, he compares the computed $h_{B}$ with the second item of the received message, if equal, he believes that $B$ has obtained the same session key as his own and then sends $h_{A}$ to $B$.

Step 3. $A \rightarrow B: h_{A}$ where $h_{A}$ is used by $A$ to inform his knowledge of $s k$ to $B$.

In the protocol, $A$ needs to perform only one SKOWHF computation, and so does $B$.

Scenario 2. A and B don't share a common key but both are under the control of $S$. (See also Figure 2.)

Involved key servers: $S$

- According to KER1

Step 1. $A \rightarrow B: n_{A}$

Since $S$ is the only intermediate server between $A$ and $B$, there are two confidentiality trunks $B$ (c) $\rightarrow S$ and $S$ (c) $\rightarrow A$ and two integrity trunks $B(\mathrm{i}) \rightarrow S$ and $S(\mathrm{i}) \rightarrow A$ should be established first to construct the required confidentiality and authentication channels.

Step 2. $B \rightarrow S: n_{B}, f\left(M K_{B},\left\{n_{B}\right\}\right) \oplus\{s k, y\}, I D_{A}, n_{A}, f\left(M K_{B}\right.$, $\left.\left\{s k, y, I D_{A}, n_{A}\right\}\right)$

As $M K_{B}$ is a common key between $B$ and $S$, we can regard $B$ 's identity $I D_{B}$ as being implicitly contained in the use of $M K_{B}$, i.e. it could be omitted.

Step 3. $S \rightarrow A: n_{S}, f\left(M K_{A},\left\{n_{S}\right\}\right) \oplus\{s k, y\}, f\left(M K_{A},\left\{s k, y, I D_{B}, n_{A}\right\}\right)$ 


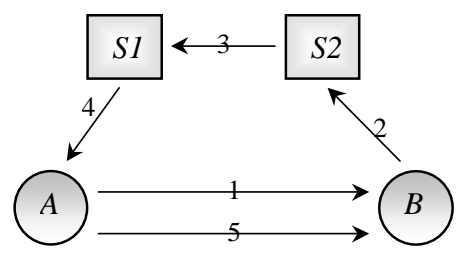

Figure 3. KEP steps of scenario 3.

Note that $I D_{B}$ is not transmitted in clear mode in the flow. Obviously, $A$ should know the identity of $B$.

Step 4. $A \rightarrow B: y$

In this protocol, $A$ and $B$ need to respectively perform two SKOWHF computations while $S$ needs four.

- According to KER2

This could be regarded as the simplest instance of case II of KER2. And, we will go into a more detail about the protocol. Similarly, $s k$ is generated by computing the SKGF: $\left\{s k, h_{A}, h_{B}\right\}=f\left(M K_{B},\left\{n_{A}, n_{B}, I D_{A}\right\}\right)$. Note that $I D_{A}$ should be included in the input of $f$. The protocol can be briefly expressed as shown below.

Step 1. $A \rightarrow B: n_{A}$

Step 2. $B \rightarrow S: I D_{A}, n_{A}, n_{B}, h_{B}$

Step 3. $S \rightarrow A: n_{S}, f\left(M K_{A},\left\{n_{S}\right\}\right) \oplus\left\{s k, h_{A}\right\}, f\left(M K_{A},\left\{s k, h_{A}, I D_{B}, n_{A}\right\}\right)$

Step 4. $A \rightarrow B: h_{A}$

Upon receiving this request, $B$ generates $n_{B}$ and then computes the SKGF to derive $\left\{s k, h_{A}\right\}$. It should be emphasized that $I D_{A}$ should be included in the parameter for $f$ so that $B$ can be protected from being fooled into believing the impersonator of $A$. On receiving the request from $B$ in flow $2, S$ also performs the SKGF to derive $\{s k$, $\left.h_{A}\right\}$ as $B$ does. Then, he compares the $h_{A}$ retrieved from flow 2 with the computed one. Once unequal, $S$ quits the protocol. Otherwise, $S$ transmits $\left\{s k, h_{A}, h_{B}\right\}$ to $A$ through a confidentiality channel and an authentication channel. Next, $A$ can verify $s k$ and recognize $B$ 's knowledge of $s k$ by checking the validity of the received messages. If correct, $A$ sends $h_{A}$ to $B$. Then, $B$ can recognize $A$ 's knowledge of $s k$ by comparing the $h_{A}$ retrieved from flow 4 and the one he holds.

We can see that $A$ authenticates $S$ as well as $B$, and $B$ authenticates $S$ as well as $A$. However, $S$ authenticate neither $A$ nor $B$. In total, $A$ needs to perform two SKOWHF computations, $B$ needs one SKOWHF computation and $S$ needs three SKOWHF computations.

Scenario 3. A and B don't share a common key. But their

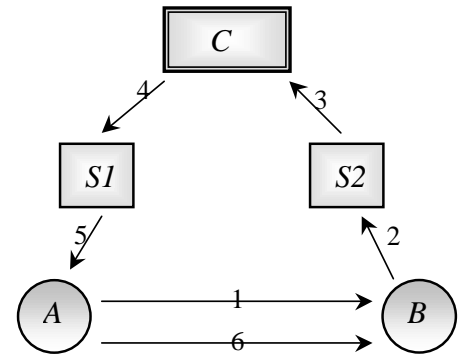

Figure 4. KEP steps of scenario 4.

key servers $S 1$ and $S 2$ have shared a common key $C K_{S I S 2}$. (See also Figure 3.)

Involved key servers: $S 2, S 1$

- According to KER1

As $S 2$ and $S 1$ are the intermediate servers between $A$ and $B$, there are three confidentiality trunks $B$ (c) $\rightarrow S 2, S 2$ (c) $\rightarrow S I$ and $S I$ (c) $\rightarrow A$ and three integrity trunks $B(\mathrm{i}) \rightarrow$ $S 2, S 2(\mathrm{i}) \rightarrow S 1$ and $S 1(\mathrm{i}) \rightarrow A$ should be established first to construct the need confidentiality and authentication channels.

Step 1. $A \rightarrow B: n_{A}$

Step 2. $B \rightarrow S 2: n_{B}, f\left(M K_{B},\left\{n_{B}\right\}\right) \oplus\{s k, y\}, I D_{A}, n_{A}, f\left(M K_{B}\right.$, $\left.\left\{s k, y, I D_{A}, n_{A}\right\}\right)$

Step 3. $S 2 \rightarrow S 1: n_{S 2}, f\left(C K_{S I S 2},\left\{n_{S 2}\right\}\right) \oplus\{s k, y\}, I D_{A}, I D_{B}$, $n_{A}, f\left(C K_{S I S 2},\left\{s k, y, I D_{A}, I D_{B}, n_{A}\right\}\right)$

Step 4.SI $\rightarrow A: n_{S l}, f\left(M K_{A},\left\{n_{S I}\right\}\right) \oplus\{s k, y\}, f\left(M K_{A},\left\{s k, y, I D_{B}, n_{A}\right\}\right)$

Step 5. $A \rightarrow B: y$

- According to KER2

This protocol simply needs to establish two sets of secure trunks should be established, i.e., $S 2$ (c) $\rightarrow S 1$ and $S 1$ $(\mathrm{c}) \rightarrow A$, and $S 2(\mathrm{i}) \rightarrow S 1$ and $S 1(\mathrm{i}) \rightarrow A$.

SKGF: $\left\{s k, h_{A}, h_{B}\right\}=f\left(M K_{B},\left\{n_{A}, n_{B}, I D_{A}\right\}\right)$

Step 1. $A \rightarrow B: n_{A}$

Step 2. $B \rightarrow S 2: I D_{A}, n_{A}, n_{B}, h_{B}$

Step 3. $S 2 \rightarrow S 1: n_{S 2}, f\left(C K_{S I S 2},\left\{n_{S 2}\right\}\right) \oplus\left\{s k, h_{A}\right\}, I D_{A}, I D_{B}$, $n_{A}, f\left(C K_{S I S 2},\left\{s k, h_{A}, I D_{A}, I D_{B}, n_{A}\right\}\right)$

Step 4. SI $\rightarrow A: n_{S I}, f\left(M K_{A},\left\{n_{S I}\right\}\right) \oplus\left\{s k, h_{A}\right\}, f\left(M K_{A},\{s k\right.$, $\left.\left.h_{A}, I D_{B}, n_{A}\right\}\right)$

Step 5. $A \rightarrow B: h_{A}$

Scenario 4. Neither A and B nor their key servers $S 1$ and $S 2$ have shared a common key. However, S1 and S2 are under the control of $C$. (See also Figure 4.)

Involved key servers: $S 2, C, S 1$

- According to KER1

Step 1. $A \rightarrow B: n_{A}$

Step 2. $B \rightarrow S 2: n_{B}, f\left(M K_{B},\left\{n_{B}\right\}\right) \oplus\{s k, y\}, I D_{A}, n_{A}, f\left(M K_{B}\right.$, $\left.\left\{s k, y, I D_{A}, n_{A}\right\}\right)$ 
Step 3. $S 2 \rightarrow C: n_{S 2}, f\left(M K_{S 2},\left\{n_{S 2}\right\}\right) \oplus\{s k, y\}, I D_{A}, I D_{B}, n_{A}$, $f\left(M K_{S 2},\left\{s k, y, I D_{A}, I D_{B}, n_{A}\right\}\right)$

Step 4. $C \rightarrow S 1: n_{C}, f\left(M K_{S l},\left\{n_{C}\right\}\right) \oplus\{s k, y\}, I D_{A}, I D_{B}, n_{A}$, $f\left(M K_{S I},\left\{s k, y, I D_{A}, I D_{B}, n_{A}\right\}\right)$

Step 5.S1 $\rightarrow A: n_{S 1}, f\left(M K_{A},\left\{n_{S 1}\right\}\right) \oplus\{s k, y\}, f\left(M K_{A},\left\{s k, y, I D_{B}, n_{A}\right\}\right)$ Step 6. $A \rightarrow B: y$

- According to KER2

SKGF: $\left\{s k, h_{A}, h_{B}\right\}=f\left(M K_{B},\left\{n_{A}, n_{B}, I D_{A}\right\}\right)$

Step 1. $A \rightarrow B: n_{A}$

Step 2. $B \rightarrow S 2: I D_{A}, n_{A}, n_{B}, h_{B}$

Step 3. S2 $\rightarrow C: n_{S 2}, f\left(M K_{S 2},\left\{n_{S 2}\right\}\right) \oplus\left\{s k, h_{A}\right\}, I D_{A}, I D_{B}$, $n_{A}, f\left(M K_{S 2},\left\{s k, h_{A}, I D_{A}, I D_{B}, n_{A}\right\}\right)$

Step 4. $C \rightarrow S 1: n_{C}, f\left(M K_{S I},\left\{n_{C}\right\}\right) \oplus\left\{s k, h_{A}\right\}, I D_{A}, I D_{B}, n_{A}$, $f\left(M K_{S l},\left\{s k, h_{A}, I D_{A}, I D_{B}, n_{A}\right\}\right)$

Step 5. $S 1 \rightarrow A: n_{S 1}, f\left(M K_{A},\left\{n_{S 1}\right\}\right) \oplus\left\{s k, h_{A}\right\}, f\left(M K_{A},\{s k\right.$, $\left.\left.h_{A}, I D_{B}, n_{A}\right\}\right)$

Step 6. $A \rightarrow B: h_{A}$

\subsection{Analysis}

The number of required SKOWHF computations by each constituent in all the four scenarios is depicted in Table 1.

\begin{tabular}{|c|c|c|c|c|c|c|c|c|}
\hline \multirow{2}{*}{$\begin{array}{l}\text { Appr. } \\
\text { Scen. }\end{array}$} & \multicolumn{4}{|c|}{ The First Approach } & \multicolumn{4}{|c|}{ The Second Approach } \\
\hline & $A$ & $B$ & $\begin{array}{c}\text { Sever } \\
\text { of } B\end{array}$ & $\begin{array}{l}\text { other } \\
\text { Server }\end{array}$ & $A$ & $B$ & $\begin{array}{l}\text { Sever } \\
\text { of } B\end{array}$ & $\begin{array}{l}\text { other } \\
\text { Server }\end{array}$ \\
\hline 1 & 2 & 2 & - & - & 1 & 1 & - & - \\
\hline 2 & 2 & 2 & 4 & - & 2 & 1 & 3 & - \\
\hline 3 & 2 & 2 & 4 & 4 & 2 & 1 & 3 & 4 \\
\hline 4 & 2 & 2 & 4 & 4 & 2 & 1 & 3 & 4 \\
\hline
\end{tabular}

Table 1. Needed SKOWHF computations by each constituent in the four scenarios.

From the table, we know that the protocols of the second approach demands fewer computations. It may be an advantage of the second approach over the first approach especially when computation should be performed in a light-weight device such as a smart card. In contrast, the first approach provides more flexibility for session key selection because the session key can be chosen to have a specific structure. However, it is not obvious that the session key should have this feature.

\section{Conclusion}

A hierarchical KEP suitable for large scale networks is constructed based on SKOWHF. With hierarchical structure of key servers, the concept of using secure trunks to construct secure channels is practical and can also be applied to any system employing cryptographic techniques. It should be emphasized that no intermediate key server needs to authenticate the received messages. Instead, only the expected recipient (end user) should authenticate the messages. It is the reason why the authentication trunk is not discussed in this paper. Conscious reader may find that the sequence of protocol steps in each scenario forms a loop-around pattern. However, either proving its optimization or contriving another better design is a topic for our further investigation.

\section{References}

[1] R. Molva, D. Samfat, and G. Tsudik, "Authentication of Mobile Users," IEEE Network, 26-34, Mar./Apr. 1994.

[2] L. Gong, "Using one-way functions for Authentication," ACM CCR, 19(5): 8-11, Oct. 1989.

[3] J.A. Bull, L. Gong, and K.R. Sollins, "Towards security in an open systems federation," in Y. Deswarte, G. Eizenberg, and J.-J. Quisquater (Eds.): Computer security - ESORICS 92', Lect. Notes Comput. Sci., 648: 3-20, 1992.

[4] A. Mathuria, "Addressing weaknesses of two cryptographic protocols of Bull, Gong and Sollins," Elect. Lett., 31: 15431544, 1995.

[5] R. Bird, I. Gopal, A. Herzberg, P. Janson, S. Kutten, R. Molva, and M. Yung, "Systematic design of a family of attack-resistant authentication protocols," IEEE J. Select. Areas Commun., 11: 679-693, June, 1993.

[6] R. Bird, I. Gopal, A. Herzberg, P. Janson, S. Kutten, R. Molva, and M. Yung, "The KryptoKnight Family of LightWeight Protocols for Authentication and Key Distribution," IEEE/ACM Trans. on Networking, 3(1): 31-41, Feb. 1995.

[7] T.A. Berson, L. Gong, and T.M.A. Lomas, "Secure, keyed, and collisionful hash functions," Technical report SRI-CSL94-08, Computer Science Laboratory, SRI International Menlo Park, CA., May 1994.

[8] C. Boyd, A.Mathuria, "Systematic design of key establishment protocols based on one-way functions," IEE Proc.Comput. Digit. Tech., 144(2): 93-99, Mar. 1997.

[9] T. Hwang and W.-C. Ku, "Reparable Key Distribution Protocols for Internet Environment," IEEE Trans. on Commun., 43(5): 1947-1949, May 1995.

[10]R. Rivest, “The MD5 Message Digest Algorithm," Internet Draft, July 1991.

[11]National Institute of standards and Technology, "Secure Hash Standard,“ NIST FIPS PUB 180, U.S. Department of Commerce, Draft, 1993.

[12]R. C. Merkle, “ A Fast Software One-Way Hash Function, “ Journal of Cryptology, 3(1): 43-58, 1990. 